\title{
RISQUE DE DÉCÈS ET RISQUE DE RUINE RÉFLEXIONS SUR LA MESURE DU RISQUE DE RUINE
}

\author{
Par Marc-Henri Amsler
}

Lausanne

RÉSUMÉ

L'article propose pour juger du risque de déséquilibre d'un portefeuille d'assurance une mesure où interviennent trois caractéristiques essentielles du risque: la probabilité de ruine, l'amplitude de la ruine et l'éloignement de l'époque d'une ruine éventuelle. Cette mesure n'est guère accessible à l'analyse mathématique mais calculable sans grands problèmes par un PC. L'auteur présente quelques exemples théoriques et pratiques.

\section{SUMMARY}

The paper proposes a three parameter measure by which the risk of a portfolio can be assessed. The parameters are: the probability of ruin, the severity of ruin (i.e. the amount of the deficit when ruin occurs) and the time of ruin. This type of analysis does not lend itself to closed form solutions, but it can be easily carried out on a PC. The author presents some theoretical and practical examples.

\section{RISQUE DE DÉCÈS ET RISQUE DE RUINE, PLUS QU'UNE ANALOGIE}

Le décès d'un assuré ainsi que la ruine d'une compagnie d'assurance (une ruine au sens mathématique!) sont assurément deux phénomènes hautement actuariels. Pour beaucoup d'actuaires néanmoins ils relèvent d'horizons fort éloignés!

Le risque de décès, étudié depuis longtemps, observé, mesuré, mis en tables depuis plus de cent ans, comparé, mathématisé dans un ensemble de formules devenues classiques, constitue la base même de la science et de la pratique actuarielle.

Le risque de ruine, d'approche plutôt spéculative, non observable, non mesurable dans la vie économique, modélisé en des formules parfois ésotériques, peu utilisable dans la pratique, est l'un des champs d'étude de prédilection de la théorie actuarielle.

Et pourtant, en ce qui touche leur structure, les deux risques, le décès et la ruine, possèdent un point essentiel en commun. Ils concernent tous deux des "systemes" vivants, susceptibles de perdre au cours du temps et de façon aléatoire justement leur qualité de "vivant». 


\section{UNE SEULE FORMULE POUR LA MESURE DES DEUX RISQUES?}

Depuis fort longtemps les actuaires ont appris à mesurer, économiquement parlant, le risque de décès: les $q_{x}$ fixent la probabilité de la réalisation du risque, le contrat d'assurance précise la prestation en capital $C_{t}$ en cas de décès à l'époque $t$, enfin le facteur d'escompte $v^{t}$ tient compte d'un laps de temps plus ou moins long séparant le présent du futur incertain. Leur formule est, en utilisant le langage international:

Mesure du risque de décès:

$$
{ }_{n} A_{x}=\sum_{t=1}^{n}{ }_{t-111} q_{x} \cdot C_{t} \cdot v^{t}
$$

L'équilibre technique à long terme de l'assurance-vie est acquis notamment par des formules établies sur des raisonnements de ce type.

Qu'en est-il du risque de ruine?

Le risque de ruine a aussi son histoire. Au début du siècle, les théoriciens de la théorie des jeux ont orienté les actuaires vers la notion de ruine des joueurs et de probabilité de cette ruine. Et l'on se mit à étudier ainsi, pour le joueur-assureur, la probabilité de perdre sa mise initiale, en un mot «de se ruiner». Fin $\mathrm{XX}^{\mathrm{e}}$ siècle, il faut se rendre à l'évidence: la science n'a toujours pas fourni un moyen d'appréhender cette probabilité dans des cas un peu généraux, autrement que par des estimateurs.

Vu la difficulté de développer une théorie susceptible de déterminer un peu confortablement la probabilité de ruine, on peut se demander si, pour mesurer le risque de déséquilibre financier, le choix exclusif de la probabilité de la ruine a été judicieux. Tentons de nous distancer de ce choix et, pour mesurer le risque de ruine, essayons de nous inspirer de la manière avec laquelle le risque de décès a été mathématisé: utilisons ce mélange judicieux des trois éléments du risque, la probabilité de l'événement, le coût du «sinistre» et l'effet du temps. Définissons donc la mesure du risque de ruine pendant une période de $n$ années par l'expression de la forme suivante (semblable à celle du risque de décès, signalée ci-dessus):

Mesure du risque de ruine:

$$
{ }_{n} A_{x}=\sum_{t=1}^{n}{ }_{t-1 \mid 1} q_{x} \cdot E\left(\left|R_{t}\right|\right) \cdot v^{t}
$$

une expression dans laquelle:

$x \quad$ serait un élément caractérisant l'état initial du portefeuille (au même titre que l'âge $x$ caractérise l'état initial de l'assuré)

${ }_{t-1 \mid 1} q$ serait la probabilité (comme en assurance-vie) de la ruine dans l'année de rang $t$,

$E\left(\left|R_{l}\right|\right) \quad$ l'espérance mathématique du montant de la ruine si elle intervient en $t$

et $v^{t} \quad$ le facteur d'escompte 
Il faut probablement renoncer actuellement à vouloir exprimer par l'analyse mathématique la valeur de la mesure ${ }_{n} A_{x}$ en fonction des caractéristiques techniques des portefeuilles. Par contre il n'est pas insensé de vouloir chercher à utiliser les ressources de l'ordinateur pour déterminer, par voie numérique, la valeur de la mesure ${ }_{n} A_{x}$.

Les lignes qui suivent montrent que par le truchement des ordinateurs et notamment des PC disponibles actuellement sur le marché il est possible de déterminer numériquement une mesure du risque de ruine qui ait exactement la forme (2) ci-dessus, c'est-à-dire le même contenu économique que celle que l'actuaire-vie utilise pour juger du risque lié à l'assurance de capital en cas de décès. L'élément $x$ de la formule (2) caractérisant l'état initial du risque de ruine sera la provision de fluctuation initiale du portefeuille.

Une remarque fondamentale s'impose: si l'on choisit la probabilité de la ruine pour mesurer le risque de ruine et pour décider du niveau de stabilité financière d'un portefeuille, il est nécessaire de fixer un "niveau acceptable» pour cette probabilité. La limite entre l'acceptable et l'inacceptable, où se situe-t-elle? Ne pourrait-on pas penser être mieux à même de trancher la question si l'on pouvait disposer d'une mesure qui puisse s'exprimer en francs, DM ou en Dollars et être comparée à d'autres éléments économiques du portefeuille? On disposerait alors d'une mesure «palpable» permettant de juger si le risque de ruine est économiquement supportable ou non? La formule (2) ci-dessus propose une mesure du risque de ruine de ce type.

La mesure du risque de ruine selon la formule (2) se justifie d'elle-même: elle représente simplement l'espérance mathématique actualisée à l'origine des temps de l'apport financier dont il serait nécessaire de disposer pour « remettre à zéro" la provision de fluctuation si celle-ci devait devenir négative.

L'actuaire-mathématicien regrettera assurément que l'on propose d'utiliser pour juger du risque de déséquilibre financier d'un portefeuille une mesure qui ne s'exprime pas par des formules du langage mathématique. Les ordinateurs existent et l'actuaire se doit d'utiliser actuellement et l'approche mathématique théorique et l'approche algorithmique numérique pour perfectionner ses techniques et son art.

Une évolution semblable s'est faite dans le domaine de l'assurance sur la vie: de Moivre, Gompertz et Makeham ont cherché à exprimer les probabilités de décès ou l'ordre des vivants par des formules mathématiques. Par cette approche, ces pioniers de la science actuarielle ont mis sur pied p. ex. la théorie de l'âge central d'un groupe de personnes. Mais il a fallu rapidement accepter la réalité : les fonctions $q_{x}$ et $l_{x}$ de même que les valeurs actuelles usuelles ne peuvent pas s'exprimer par des formules mathématiques générales si l'on veut pouvoir s'adapter au monde réel. Et depuis fort longtemps les actuaires-vie déterminent l'ordre des vivants et les valeurs actuelles non par des formules mathématiques mais par l'algorithme récursif:

$$
l_{x}-d_{x}=l_{x+1}
$$

et par des sommations de nombres de commutations (donc des opérations purement numériques). 


\section{LE MODÈLE ALGORITHMIQUE}

Le processus aléatoire classique décrivant l'évolution d'un portefeuille d'assurance est défini comme suit:

L'institution d'assurance dispose à l'origine des temps d'une provision de fluctuation $x$, encaisse chaque année un volume de primes $P$, doit faire face à des prestations d'assurance de montant $S$ (aléatoire) par année, la prime $P$ comprenant une marge de sécurité positive par rapport au coût moyen $E(S)$ des sinistres. Le protefeuille est stationnaire en ce sens que le volume des primes annuelles $P$ est constant et les charges annuelles des sinistres $S$ des variables indépendantes et identiquement distribuées (iid).

La formule de récurrence reliant deux valeurs consécutives de la provision de fluctuation (aléatoire) $R_{t}$ est :

$$
\begin{aligned}
R_{0}=x & \\
R_{t}+P-S & =R_{t+1} \quad t=0,1,2,3, \ldots
\end{aligned}
$$

On notera au passage la ressemblance de cette formule de récurrence (4) avec celle de l'assurance-vie (3).

Pour satisfaire aux exigences du calcul numérique (et sans perte essentielle de généralité), on supposera que la charge annuelle $S$ des sinistres prend des valeurs multiples d'une unité monétaire donnée et qu'elle est bornée (cette dernière hypothèse étant d'ailleurs satisfaite dans la réalité).

Le modèle choisi est donc discret en valeur et dans le temps.

La ruine intervient au jour du bilan pour lequel $R_{t}$ devient négatif pour la $1^{\mathrm{re}}$ fois $\left(1^{\mathrm{re}}\right.$ ruine).

En un moment d'observation $t$ quelconque ( $t$ entier) le portefeuille peut se trouver dans l'un ou l'autre des 3 états suivants:

- état $\mathrm{A}$ : n'avoir jamais été en situation de ruine $\left(R_{t}\right.$ toujours positif jusqu'en $t, t$ compris),

- état B: être tombé en situation de ruine juste en $t(\ldots) 1^{\mathrm{rc}}$ ruine),

- état $C$ : être tombé en situation de ruine à une date antérieure.

Ces 3 états représentent la totalité des états possibles d'un portefeuille à l'époque $t$.

La succession des états peut être représentée par le graphe suivant, chaque flèche indiquant une évolution possible de l'état du portefeuille au cours d'une année:

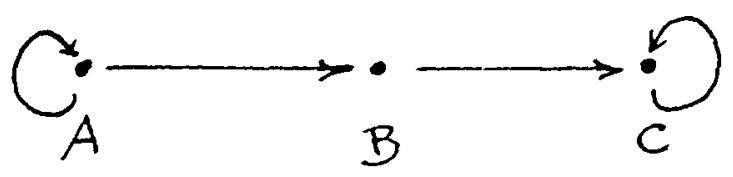

$\mathrm{Vu}$ les hypothèses faites sur le modèle, les distributions des variables $R_{t}$ $(t=1,2,3, \ldots)$ s'obtiennent par convolutions successives de distributions discrètes. Le processus de calcul de ces distributions est élémentaire, il doit 
simplement enregistrer les probabilités obtenues pour chacune des valeurs possibles de $R_{t}$ séparément selon les 3 états $\mathrm{A}$, B et C.

La détermination numérique de la mesure du risque de ruine telle que proposée par la formule (2) exige la connaissance aux époques $t=1,2,3, \ldots, n$ :

- de la probabilité du déplacement du portefeuille de l'état A vers l'état B, soit la grandeur ${ }_{t-1 \mid 1} q_{x}$,

- des probabilités des valeurs de $R_{t}$ liées à l'état B afin d'en tirer l'espérance mathématique $E\left(\left|R_{t}\right|\right)$,

- des valeurs successives du facteur d'escompte $v^{t}$.

Voici quelques exemples:

\section{EXEMPLES}

Nous présenterons successivement trois exemples. Tout d'abord deux «exemples d'école", non réalistes dans le cadre d'études de portefeuilles d'assurance, mais permettant de contrôler par la voie du calcul numérique plusieurs propriétés classiques fournies par la voie mathématique. Enfin un dernier exemple, cette fois-ci réel, provenant d'une modélisation d'une caisse de pension de droit suisse (d'après E. Kuhn, Bulletin de l'Association suisse des actuaires, Vol. 1986/2). Ce dernier exemple permettra, par l'introduction de diverses formes de réassurance, de juger dans un cas pratique de l'effet de ces réassurances sur la mesure du risque de ruine ${ }_{n} A_{x}$, et, de plus, de constater laquelle des 3 composantes de la mesure du risque, la probabilité de la ruine, le montant de la ruine ou le laps de temps jusqu'à une ruine éventuelle joue un rôle déterminant dans l'estimation du risque.

Les résultats des 2 premiers exemples seront présentés par des tableaux numériques de la forme:

Determination de la mesure du Risque - Portefeuille Ber604

\begin{tabular}{lcccccccc}
\hline \hline & \multicolumn{3}{c}{ Probabilités } \\
$t$ & ${ }_{t} p_{x}$ & $q_{x, t}$ & ${ }_{t-1 \mid 1 q_{x}}$ & cumul & $E\left(\left|R_{t}\right|\right)$ & $\begin{array}{c}\text { Mesure du risque } \\
1 \text { an }\end{array}$ & ${ }_{t} A_{x}$ & $v^{t}$ \\
\hline 0 & 1.00000 & .00000 & .00000 & .00000 & .00000 & .00000 & .00000 & 1.00000 \\
1 & 1.00000 & .00000 & .00000 & .00000 & .00000 & .00000 & .00000 & .95238 \\
2 & 1.00000 & .00000 & .00000 & .00000 & .00000 & .00000 & .00000 & .90703 \\
3 & 1.00000 & .02560 & .00000 & .00000 & .00000 & .00000 & .00000 & .86384 \\
4 & .97440 & .00000 & .02560 & .02560 & 1.00000 & .02560 & .02106 & .82270 \\
\hline
\end{tabular}

La signification des diverses colonnes est la suivante: (afin de souligner l'identité de structure du processus «ruine-non ruine» avec le processus «vie-décès», nous utiliserons le langage et les symboles usuels de l'assurance- 
vie; nous parlerons notamment de "portefeuille en vie» lorsque, au cours du temps, le portefeuille ne sera jamais tombé en situation de ruine).
Col. No Symbole
Signification
$1 \quad{ }_{t} p_{x} \quad$ probabilité, pour le portefeuille disposant d'une provision de fluctuation initiale de montant $x$, d'être encore «en vie» à l'époque $t$,
$2 q_{x, t} \quad$ probabilité en $t$ de la première ruine dans l'année qui suit ("en vie» en $t$, "décédé» en $t+1$, soit le $q_{x+t}$ usuel de l'assurance-vie),
$3 t_{t-1 \mid 1} q_{x}$ probabilité en $t=0$ d'une $1^{\text {re }}$ ruine (décès) exactement à l'époque $t$,
$4 \quad-\quad$ cumul de la col. 3, soit probabilité d'avoir été en situation de ruine pendant les $t$ années écoulées,
$5 E\left(\left|R_{t}\right|\right)$ espérance mathématique du montant d'une ruine en $t$,
$6-$ prime de risque de l'année de rang $t=t_{t-1 \mid 1} q_{x} \cdot E\left(\left|R_{t}\right|\right)$,
$7{ }_{t} A_{x} \quad$ mesure en $t=0$ du risque de ruine pendant le laps de temps $t$, soit le cumul actualisé en $t=0$ des primes de risque annuelles selon col. 6,
$8 v^{t} \quad$ facteur d'escompte usuel.

Pour le $3^{\mathrm{e}}$ exemple les tableaux numériques III et IV sont des extraits de tableaux de même nature établis pour les diverses formes de réassurance considérées.

Les calculs ont été réalisés sur des P.C. soit en langage APL soit en langage C, en collaboration avec le professeur François Dufresne, Université Laval, Canada.

\section{Exemple 1}

Considérons le modèle bernoullien de caractéristiques suivantes (modèle Ber604):

- valeurs possibles de la charge $S$ des sinistres:

$0 \quad 1 \quad 2$

- probabilités correspondant à ces 3 valeurs :

$$
p=0.6 \quad 0 \quad q=0.4
$$

— on a $E(S)=0.8 \quad \operatorname{Var}(S)=0.96$

- prime annuelle $P=1$ (marge de sécurité $P-E(S)=0.2$ )

- provision de fluctuation initiale $x=3$,

- actualisation au taux annuel de 5\%

Voici les résultats des calculs: 
TABLEAU 1

Determination de la mesure du Risque - Portefeuille BER604

\begin{tabular}{|c|c|c|c|c|c|c|c|c|}
\hline \multirow[b]{2}{*}{$t$} & \multirow[b]{2}{*}{$p_{x}$} & \multicolumn{3}{|c|}{ Probabilités } & \multirow[b]{2}{*}{$E\left(\left|R_{t}\right|\right)$} & \multicolumn{2}{|c|}{ Mesure du risque } & \multirow{2}{*}{$i=\underbrace{.} 0.05$} \\
\hline & & $q_{x, t}$ & $t-1 \mid 1 q_{x}$ & cumul & & 1 an & ${ }_{1} A_{x}$ & \\
\hline 0 & 1.00000 & .00000 & .00000 & .00000 & .00000 & .00000 & .00000 & 1.00000 \\
\hline 1 & 1.00000 & .00000 & .00000 & .00000 & .00000 & .00000 & .00000 & .95238 \\
\hline 2 & 1.00000 & .00000 & .00000 & .00000 & .00000 & .00000 & .00000 & .90703 \\
\hline 3 & 1.00000 & .02560 & .00000 & .00000 & .00000 & .00000 & .00000 & .86384 \\
\hline 4 & .97440 & .00000 & .02560 & .02560 & 1.00000 & .02560 & .02106 & .82270 \\
\hline 5 & .97440 & .02522 & .00000 & .02560 & .00000 & .00000 & .02106 & .78353 \\
\hline 6 & .94982 & .00000 & .02458 & .05018 & 1.00000 & .02458 & .03940 & .74622 \\
\hline 7 & .94982 & .02173 & .00000 & .05018 & .00000 & .00000 & .03940 & .71068 \\
\hline 8 & .92918 & .00000 & .02064 & .07082 & 1.00000 & .02064 & .05337 & .67684 \\
\hline 9 & .92918 & .01828 & .00000 & .07082 & .00000 & .00000 & .05337 & .64461 \\
\hline 10 & .91219 & .00000 & .01699 & .08781 & 1.00000 & .01699 & .06380 & .61391 \\
\hline 11 & .91219 & .01536 & .00000 & .08781 & .00000 & .00000 & .06380 & .58468 \\
\hline 12 & .89818 & .00000 & .01401 & .10182 & 1.00000 & .01401 & .07160 & .55684 \\
\hline 13 & .89818 & .01298 & .00000 & .10182 & .00000 & .00000 & .07160 & .53032 \\
\hline 14 & .88652 & .00000 & .01166 & .11348 & 1.00000 & .01166 & .07749 & .50507 \\
\hline 15 & .88652 & .01105 & .00000 & .11348 & .00000 & .00000 & .07749 & .48102 \\
\hline 16 & .87672 & .00000 & .00979 & .12328 & 1.00000 & .00979 & .08198 & .45811 \\
\hline 17 & .87672 & .00947 & .00000 & .12328 & .00000 & .00000 & .08198 & .43630 \\
\hline 18 & .86842 & .00000 & .00830 & .13158 & 1.00000 & .00830 & .08543 & .41552 \\
\hline 19 & .86842 & .00818 & .00000 & .13158 & .00000 & .00000 & .08543 & .39573 \\
\hline 20 & .86132 & .00000 & .00710 & .13868 & 1.00000 & .00710 & .08811 & .37689 \\
\hline 21 & .86132 & .00710 & .00000 & .13868 & .00000 & .00000 & .08811 & .35894 \\
\hline 22 & .85521 & .00000 & .00612 & .14479 & 1.00000 & .00612 & .09020 & .34185 \\
\hline 23 & .85521 & .00620 & .00000 & .14479 & .00000 & .00000 & .09020 & .32557 \\
\hline 24 & .84990 & .00000 & .00531 & .15010 & 1.00000 & .00531 & .09184 & .31007 \\
\hline 25 & .84990 & .00545 & .00000 & .15010 & .00000 & .00000 & .09184 & .29530 \\
\hline
\end{tabular}

On remarquera en particulier:

la réduction progressive des probabilités de ruine (col. 2 et 3 ),

- la constance de $E\left(\left|R_{t}\right|\right)$, col. 5, évidente, puisque dans ce modèle le montant d'une ruine est toujours égal à 1 ,

- l'effet de l'actualisation (à 5\%): sans escompte $\left(v^{t}=1\right.$ et puisque $E\left(\left|R_{t}\right|\right)=1$ pour tous les $t$ ) la mesure du risque de ruine ${ }_{t} A_{x}$ correspondant à une période $t$ quelconque est égale à la probabilité de ruine cumulée sur ladite période (col. 4). Avec escompte de $5 \%$ par année, la mesure du risque de ruine sur 25 ans ${ }_{25} A_{3}$ p. ex. est 0.09184 , se monte ainsi à $61,2 \%$ de la valeur sans escompte $(0.15010)$.

Il est intéressant de comparer le cumul progressif de la probabilité de ruine (col. 4) avec la valeur exacte limite donnée par la théorie mathématique pour une période infiniment longue:

$$
\text { prob. }=(q: p)^{x+1}=(0.4: 0.6)^{4}=0.19753
$$

Ainsi après 25 ans la probabilité de ruine atteint 76,0\% (et après 50 ans $92,5 \%$ ) de la valeur limite donnée par la théorie. Il faut attendre 110 ans pour que la probabilité de ruine atteigne les $99 \%$ de la valeur limite 0.19753. 


\section{Exemple 2}

Refaisons les mêmes calculs mais en partant du modèle pour lequel la charge annuelle $S$ des sinistres est une variable géométrique

$$
\operatorname{prob}(S=s)=(1-r) \cdot r^{s} \quad \text { avec } \quad r=4 / 9
$$

On a également

$$
E(S)=0.8 \quad \text { mais } \quad \operatorname{Var}(S)=1.44
$$

Pour une même provision de fluctuation initiale $x=3$, les résultats sont les suivants :

TABLEAU 2

\begin{tabular}{|c|c|c|c|c|c|c|c|c|}
\hline \multirow[b]{2}{*}{$t$} & \multirow[b]{2}{*}{$t_{x}$} & \multicolumn{3}{|c|}{ Probabilités } & \multirow[b]{2}{*}{$E\left(\left|R_{t}\right|\right)$} & \multicolumn{2}{|c|}{ Mesure du risque } & \multirow{2}{*}{$\begin{array}{c}i=0.05 \\
v^{i}\end{array}$} \\
\hline & & $q_{x, t}$ & $t-1 \mid 1 q_{x}$ & cumul & & 1 an & ${ }_{1} A_{x}$ & \\
\hline 0 & 1.00000 & .01734 & .00000 & .00000 & .00000 & .00000 & .00000 & 1.00000 \\
\hline 1 & .98266 & .02179 & .01734 & .01734 & 1.80154 & .03124 & .02975 & .95238 \\
\hline 2 & .96125 & .02200 & .02141 & .03875 & 1.80123 & .03856 & .06473 & .90703 \\
\hline 3 & .94010 & .02083 & .02114 & .05990 & 1.80122 & .03809 & .09736 & .86384 \\
\hline 4 & .92053 & .01926 & .01958 & .07947 & 1.80128 & .03527 & .12665 & .82270 \\
\hline 5 & .90280 & .01765 & .01773 & .09720 & 1.80139 & .03193 & .15166 & .78353 \\
\hline 6 & .88687 & .01613 & .01593 & .11313 & 1.80151 & .02870 & .17308 & .74622 \\
\hline 7 & .87256 & .01475 & .01430 & .12744 & 1.80166 & .02577 & .19140 & .71068 \\
\hline 8 & .85970 & .01350 & .01287 & .14030 & 1.80164 & .02318 & .20709 & .67684 \\
\hline 9 & .84809 & .01239 & .01161 & .15191 & 1.80159 & .02092 & .22057 & .64461 \\
\hline 10 & .83758 & .01140 & .01051 & .16242 & 1.80152 & .01894 & .23219 & .61391 \\
\hline 11 & .82803 & .01052 & .00955 & .17197 & 1.80146 & .01721 & .24225 & .58468 \\
\hline 12 & .81932 & .00973 & .00871 & .18068 & 1.80140 & .01569 & .25099 & .55684 \\
\hline 13 & .81135 & .00902 & .00797 & .18865 & 1.80134 & .01436 & .25861 & .53032 \\
\hline 14 & .80403 & .00838 & .00732 & .19597 & 1.80128 & .01318 & .26526 & .50507 \\
\hline 15 & .79729 & .00780 & .00674 & .20271 & 1.80123 & .01214 & .27110 & .48102 \\
\hline 16 & .79107 & .00728 & .00622 & .20893 & 1.80118 & .01121 & .27623 & .45811 \\
\hline 17 & .78531 & .00681 & .00576 & .21469 & 1.80113 & .01038 & .28076 & .43630 \\
\hline 18 & .77996 & .00638 & .00535 & .22004 & 1.80108 & .00963 & .28476 & .41552 \\
\hline 19 & .77498 & .00599 & .00498 & .22502 & 1.80103 & .00896 & .2883 & .39573 \\
\hline 20 & .77034 & .00563 & .00464 & .22966 & 1.80098 & .00836 & .29146 & .37689 \\
\hline 21 & .76601 & .00530 & .00433 & .23399 & 1.80094 & .00781 & .29426 & .35894 \\
\hline 22 & .76195 & .00499 & .00406 & .23805 & 1.80089 & .00731 & .29676 & .34185 \\
\hline 23 & .75815 & .00471 & .00381 & .24185 & 1.80085 & .00685 & .29899 & .32557 \\
\hline 24 & .75457 & .00446 & .00357 & .24543 & 1.80081 & .00644 & 30099 & .31007 \\
\hline 25 & .75121 & .00000 & .00336 & .24879 & 1.80077 & .00606 & .30277 & .29530 \\
\hline
\end{tabular}

Détermination de la mesure du RisQue - Portefeullle GEOMEO8M12

\section{On remarquera en particulier que}

- les probabilités de ruine ${ }_{t-1 \mid 1} q_{x}$ croissent pendant les 2 premières années puis décroissent lentement,

- $E\left(\left|R_{t}\right|\right)$ est constant. L'approche mathématique établit en effet que dans ce modèle particulier le montant de la ruine a la même loi de distribution que la charge $S$ elle-même. Vu que le montant de la ruine accepte des valeurs à 
partir de 1 et non de 0 (la situation $R=0$ ne représente pas une « ruine» au sens actuariel), l'espérance mathématique $E\left(\left|R_{t}\right|\right)$, pour tous les temps $t$, est égale à $1+E(S)=1.8$. La petite imprécision dans les dernières décimales provient du fait que pour le calcul numérique il a fallu tronquer la distribution (avantage et désavantage du calcul numérique!). La troncature a été faite comme suit:

$$
\begin{aligned}
& \text { probabilités de } S \text { jusqu'à } S=10 \text { selon formule } \\
& \text { prob }(S=11)=0 \\
& \operatorname{prob}(S=12)=0.0001336571821
\end{aligned}
$$

de façon à obtenir une espérance mathématique très proche de 0.8 ,

- la convergence de la probabilité de ruine sur $t$ années vers sa limite pour une période infinie, donnée par la théorie mathématique:

$$
\text { prob. }=(r: 1-r)^{x+2}=0.32768 \quad(x=3)
$$

La probabilité de ruine pendant la période considérée de 25 ans est, selon le tableau:

$$
\text { prob. sur } 25 \text { ans }=0.24879
$$

On est encore loin (à $75,9 \%$ ) de la valeur limite!

\section{Exemple 3}

Le portefeuille est celui d'une caisse de pensions comprenant 236 assurés actifs, d'âge moyen 36,48 . Les sommes sous le risque - calculées pour chaque assuré - varient entre -50000 et +680000 Frs.s. Le nombre moyen annuel des sinistres est 0.917 .

La charge annuelle $S$ est une variable de Poisson pondérée.

Dans la présente étude, l'unité de calcul a été choisie égale à Frs.s. 20000.

On a

$$
E(S)=6.3640 \quad \operatorname{Var}(S)=105.0
$$

Pour les calculs numériques, objet des tableaux ci-dessous, on a choisi comme suit les derniers paramètres du portefeuille:

Prime annuelle totale du portefeuille $\quad P=8(=160000)$

Provisions de fluctuation initiale $\quad x=0,10,20$ et 30

Actualisation au taux annuel d'intérêt $i=5 \%$

Durée de l'évolution du processus $\quad n=20$ ans.

Le calcul de la mesure ${ }_{n} A_{x}$ et de ses éléments a été effectué tout d'abord pour le portefeuille original, donc sans réassurance, puis pour le portefeuille pour propre compte après réassurance en Stop-loss et en Excess-of-loss dans le but de juger des effets d'une réassurance sur la mesure du risque de ruine ${ }_{n} A_{x}$ proposée.

Voici les résultats des calculs: 
TABLEAU 3

PoRTEFEUILle: CAISSE DE PENSIONS ALPHA 1

EVOLUTION PENDANT UNE PÉRIODE DE 20 ANS

\begin{tabular}{|c|c|c|c|c|c|c|c|c|c|}
\hline \multirow[b]{2}{*}{ Reass. } & \multirow[b]{2}{*}{ Plein } & \multirow[b]{2}{*}{$E(S)$} & \multirow[b]{2}{*}{$\operatorname{Var}(S)$} & \multirow[b]{2}{*}{$P$} & \multirow[b]{2}{*}{$P / E(S)$} & \multicolumn{4}{|c|}{ Prov. de fluctuation initiale } \\
\hline & & & & & & $x=0$ & $x=10$ & $x=20$ & $x=30$ \\
\hline \multicolumn{10}{|c|}{ Probabilités de ruine } \\
\hline - & - & 6.364 & 105.0 & 8 & 1.257 & .65826 & .48981 & .34785 & .24062 \\
\hline $\mathrm{XL}$ & 20 & 5.823 & 78.5 & 7 & 1.202 & .69333 & .50435 & .33648 & .22225 \\
\hline SL & 20 & 5.223 & 51.8 & 6 & 1.149 & .74032 & .51856 & .31284 & .18032 \\
\hline $\mathrm{XL}$ & 10 & 4.282 & 34.4 & 5 & 1.168 & .71729 & .42008 & .22472 & .11254 \\
\hline SL & 10 & 3.546 & 18.0 & 4 & 1.128 & .75835 & .36447 & .13543 & .04201 \\
\hline \multicolumn{10}{|c|}{ Mesure du risque ${ }_{n} A_{x}$ sans escompte } \\
\hline- & - & 6.364 & 105.0 & 8 & 1.257 & 7.6656 & 5.1510 & 3.5627 & 2.4807 \\
\hline XL & 20 & 5.823 & 78.5 & 7 & 1.202 & 6.6779 & 4.1135 & 2.8390 & 1.8429 \\
\hline SL & 20 & 5.223 & 51.8 & 6 & 1.149 & 5.2468 & 2.5710 & 1.6255 & 0.9545 \\
\hline $\mathrm{XL}$ & 10 & 4.282 & 34.4 & 5 & 1.168 & 4.2637 & 2.2461 & 1.1847 & 0.5917 \\
\hline SL & 10 & 3.546 & 18.0 & 4 & 1.128 & 2.8919 & 0.9857 & 0.3800 & 0.1165 \\
\hline \multicolumn{10}{|c|}{ Mesure du risque ${ }_{n} A_{x}$ avec escompte au taux de $i=.0500$} \\
\hline - & - & 6.364 & 105.0 & 8 & 1.257 & 6.5820 & 4.1368 & 2.6654 & 1.7442 \\
\hline XL & 20 & 5.823 & 78.5 & 7 & 1.202 & 5.7599 & 3.2485 & 2.0629 & 1.2412 \\
\hline SL & 20 & 5.223 & 51.8 & 6 & 1.149 & 4.6162 & 1.9902 & 1.1166 & .5938 \\
\hline XL & 10 & 4.282 & 34.4 & 5 & 1.168 & 3.6966 & 1.6876 & .7870 & .3565 \\
\hline SL & 10 & 3.546 & 18.0 & 4 & 1.128 & 2.5709 & .6872 & .2220 & .0599 \\
\hline
\end{tabular}

Le premier tiers du tableau donne, dans les différents cas de figure, les probabilités d'une ruine pendant les 20 années considérées.

Le second tiers indique les valeurs de la mesure ${ }_{n} A_{x}$ si l'on ne tient pas compte du phénomène de l'escompte $(i=0)$.

Le troisième tiers donne les valeurs $\operatorname{de}_{n} A_{x}$ en tenant compte du phénomène de l'escompte, au taux de 5\% l'an.

Voici le contenu des premières colonnes du tableau:

col. 1: SL signifie «Stop-loss», et XL «Excess-of-loss»

col. 2: plein de conservation

col. 3 et 4: espérance mathématique et variance de la charge des sinistres pour propre compte

col. 5: prime annuelle $P$, marge de sécurité comprise

col. 6: le rapport $P / E$ informe sur l'importance de la marge de sécurité. Pour les formes avec réassurance, la marge a été réduite vu la réduction des fluctuations du portefeuille par suite de la réassurance.

On peut faire les constatations suivantes qui, évidemment, ne sont valables que pour l'exemple traité. Il n'est pas irréaliste de penser que ces constatations doivent probablement être également valables en ordre de grandeur pour d'autres portefeuilles «réels». 


\section{Constatations}

1. En ce qui concerne les probabilités de ruine $\left(1^{\text {er }}\right.$ tiers du tableau) on constate que les réassurances prévues n'ont pas un grand effet sur leurs valeurs numériques (si l'on excepte la variante extrême avec une provision de fluctuation initiale de $x=30$, soit de près de 4 primes annuelles!). Ce fait pourrait provenir de ce que les probabilités étant très fortes, les réassurances prévues ne permettent pas d'éviter la ruine, mais peut-être seulement les «fortes ruines», ce qui évidemment ne se voit pas dans la valeur de la probabilité seule de la ruine.

2. La prise en considération du montant de la ruine en plus de la probabilité $\left(2^{\mathrm{e}}\right.$ tiers du tableau) fournit des mesures du risque ${ }_{n} A_{x}$ (sans escompte) beaucoup plus différenciées entre la forme sans et les formes avec réassurance. Cette constatation semble confirmer l'hypothèse faite ci-dessus que la réassurance agit en priorité non sur la probabilité mais sur le montant de la ruine. La réduction de la mesure du risque de ruine ${ }_{n} A_{x}$ par suite d'une réassurance est même très forte. Ce fait permet de penser que la seule probabilité de la ruine n'est peut-être pas "le critère par excellence" pour estimer la stabilité d'un portefeuille d'assurance.

3. L'effet de l'élément "temps d'attente jusqu'à l'époque de la ruine» par l'incorporation du facteur d'escompte $v^{t}$ dans la formule de ${ }_{n} A_{x}$ peut être mesuré en comparant le $3^{\mathrm{e}}$ au $2^{\mathrm{e}}$ tiers du tableau. L'importance de la réduction donne une certaine information sur la durée de vie moyenne du portefeuille: une forte réduction indique que la ruine interviendra plutôt tardivement (si elle intervient), une faible réduction que le risque se situe dans un avenir pas trop lointain.

Vu le caractère prépondérant du montant de la ruine dans la mesure ${ }_{n} A_{x}$, il est intéressant de connaître l'évolution de l'espérance mathématique de cette ruine au cours du temps. Le tableau IV suivant en donne les valeurs numériques pour le portefeuille sans et avec les 4 réassurances considérées, sous l'hypothèse d'une provision de fluctuation initiale $x=10$.

En comparant les colonnes entre elles, on constatera en effet que la réassurance réduit fortement le montant moyen de la ruine. D'autre part, chose étrange, après quelques années d'évolution, le montant moyen de la ruine semble se stabiliser et cela aussi bien pour le portefeuille sans réassurance que pour les 4 portefeuilles pour propre compte. Est-ce là une propriété générale ou une propriété particulière du portefeuille considéré? Ce point mérite une étude plus poussée, notamment vu que pour les deux «modèles d'école» connus (cas bernoullien et géométrique) les montants de la ruine sont identiquement distribués pour tous les temps.

On se souviendra également que dans le modèle en temps continu «Poissonexponentiel », la distribution du montant de la ruine est indépendante du temps $t$, conduit donc à une espérance mathématique constante du montant de la ruine. 
TABLEAU 4

ESPERANCES MATHEMATIQUES DES MONTANTS DE LA RUINE 5 DISTRIBUTIONS, PROVISION DE FLUCTUATION $x=10$

\begin{tabular}{|c|c|c|c|c|c|}
\hline \multirow[b]{2}{*}{$t$} & \multirow{2}{*}{$\begin{array}{c}\text { Portefeuille } \\
\text { original }\end{array}$} & \multicolumn{4}{|c|}{ Avec réassurance } \\
\hline & & $X L 20$ & SL20 & XL10 & SL10 \\
\hline 1 & 10.68237 & 7.11305 & 3.57901 & 5.32897 & 1.00000 \\
\hline 2 & 10.45803 & 8.83038 & 5.56497 & 5.29275 & 1.87975 \\
\hline 3 & 10.52935 & 8.75998 & 6.30800 & 5.51549 & 3.14442 \\
\hline 4 & 10.55995 & 8.37064 & 5.63211 & 5.29166 & 3.07209 \\
\hline 5 & 10.49723 & 8.49668 & 5.09456 & 5.41297 & 2.58590 \\
\hline 6 & 10.45528 & 8.50833 & 5.43031 & 5.31850 & 3.19702 \\
\hline 7 & 10.43762 & 8.45012 & 5.47503 & 5.36327 & 2.74371 \\
\hline 8 & 10.41972 & 8.45204 & 5.29235 & 5.32769 & 2.81429 \\
\hline 9 & 10.40305 & 8.44830 & 5.31522 & 5.33747 & 2.91595 \\
\hline 10 & 10.39134 & 8.43722 & 5.35058 & 5.32552 & 2.75674 \\
\hline 11 & 10.38108 & 8.43061 & 5.33487 & 5.32804 & 2.86498 \\
\hline 12 & 10.37292 & 8.42410 & 5.30397 & 5.32068 & 2.82167 \\
\hline 13 & 10.36694 & 8.41507 & 5.30695 & 5.31771 & 2.80272 \\
\hline 14 & 10.36697 & 8.41204 & 5.30909 & 5.31334 & 2.83534 \\
\hline 15 & 10.36245 & 8.41123 & 5.29765 & 5.31477 & 2.80282 \\
\hline 16 & 10.35852 & 8.40459 & 5.29290 & 5.31304 & 2.81678 \\
\hline 17 & 10.35336 & 8.40188 & 5.29437 & 5.30811 & 2.81591 \\
\hline 18 & 10.33398 & 8.39316 & 5.28899 & 5.31085 & 2.80463 \\
\hline 19 & 10.33474 & 8.38961 & 5.28808 & 5.31062 & 2.81440 \\
\hline 20 & 10.33867 & 8.39157 & 5.28571 & 5.30717 & 2.80861 \\
\hline
\end{tabular}

\section{CONCLUSIONS}

Lorsque l'on parle «stabilité financière» d'un portefeuille d'assurance, par tradition on pense «probabilité de ruine»; il y a plus de 60 ans qu'un aiguillage, peut-être inconscient, a orienté les esprits sur cette voie. Les études de stabilité qui s'ensuivirent ont largement utilisé cet outil par excellence de l'actuaire que sont les mathématiques et notamment l'analyse mathématique. Le recours à cet outil eut le grand mérite d'exiger précision et concision dans les choix faits sur les définitions et les hypothèses sur lesquelles se sont construits peu à peu les modèles. Néanmoins, si l'on regarde la moisson des résultats, le constat est plutôt maigre. Pratiquement seuls des modèles d'école ont permis d'apporter des réponses un peu précises, ces modèles parlant de risques bernoulliens, géométriques, exponentiels ou poissoniens, de plus tablant dans la plupart des cas sur l'hypothèse d'un futur infini. Le cadre des recherches par la voie analytique ne correspond ainsi pas trop bien à celui que la réalité nous révèle.

Et c'est sur ce point précis de l'adaptation à la réalité que l'ordinateur apporte son éclairage propre: point d'analyse mathématique ni de temps infini, mais des modèles largement libérés des contraintes mathématiques, un cadre numérique et un horizon de court et moyen terme. Incapable - du moins actuellement - de s'aventurer sur les voies de l'analyse mathématique conçues par l'esprit humain, mais passé maître dans l'exécution de calculs pour lesquels 
l'homme ne montre ni engouement ni prédisposition, l'ordinateur propose ainsi une complémentarité bienvenue à l'approche purement mathématique dans l'étude des conditions de stabilité des portefeuilles d'assurance.

Le présent article propose une mesure du risque de déséquilibre financier des portefeuilles d'assurance intuitivement élémentaire et aisément accessible au calcul numérique. Cette mesure fait intervenir, outre la probabilité et ruine, l'amplitude de la ruine ainsi que l'éloignement dans le temps de l'époque d'une ruine éventuelle. Elle est conceptuellement identique à la mesure qu'utilisent chaque jour les actuaires-vie, pour estimer le risque financier le plus élémentaire connu, soit la prime unique de l'assurance de capital en cas de décès.

Un exemple - calqué sur la situation tirée de la pratique d'une caisse de pension - montre en particulier que (du moins pour cet exemple) les réassurances en excess-of-loss et en stop-loss n'ont que peu d'effet sur la probabilité de ruine du portefeuille, par contre un effet très marqué sur l'amplitude d'une ruine éventuelle (chose assez compréhensible!). D'autres exemples en cours d'élaboration révèlent les mêmes caractéristiques. Cette constatation parle ainsi en faveur de l'extension de la mesure classique du risque par sa probabilité à une mesure faisant intervenir également l'amplitude de la ruine, un élément qui a son poids dans l'estimation objective et subjective du risque.

Ce même exemple enfin - comme d'autres non publiés ici - révèle une certaine stabilité de l'espérance mathématique de l'amplitude de la ruine au cours du temps dans un horizon à moyen terme, même une tendance à converger lorsque l'horizon du temps s'éloigne de plus en plus. Ce dernier fait mérite qu'on en poursuivre l'étude.

Lausanne, le 6 décembre 1991

Marc-Henri Amsler

Ecole des H.E.C., Université, CH-1015 Lausanne. 